Pacific Journal of Mathematics

EXPLICIT PL SELF-KNOTTINGS AND THE STRUCTURE OF
PL HOMOTOPY COMPLEX PROJECTIVE SPACES 


\title{
EXPLICIT PL SELF-KNOTTINGS AND THE STRUCTURE OF PL HOMOTOPY COMPLEX PROJECTIVE SPACES
}

\author{
Douglas Meadows
}

\begin{abstract}
We show that certain piecewise-linear homotopy complex projective spaces may be described as a union of smooth manifolds glued along their common boundaries. These boundaries are sphere bundles and the glueing homeomorphisms are piecewise-linear self-knottings on these bundles. Furthermore, we describe these self-knottings very explicitly and obtain information on the groups of concordance classes of such maps.
\end{abstract}

A piecewise linear homotopy complex projective space $\widetilde{C P}^{n}$ is a compact PL manifold $M^{2 n}$ homotopy equivalent to $C P^{n}$. In [22] Sullivan gave a complete enumeration of the set of PL isomorphism classes of these manifolds as a consequence of his Characteristic Variety theorem and his analysis of the homotopy type of G/PL. In [15] Madsen and Milgram have shown that these manifolds, the index 8 Milnor manifolds, and the differentiable generators of the oriented smooth bordism ring provide a complete generating set for the torsion-free part of the oriented PL bordism ring. Hence a study of the geometric structure of these exotic projective spaces $\widetilde{C P^{n}}$, especially with regard to their smooth singularities, may extend our understanding of the PL bordism ring. This paper begins such a study in which we obtain a geometric decomposition of $\widetilde{C P}^{n}$, into (for many cases) a union of smooth manifolds glued together by PL self-knottings on certain sphere bundles. We also obtain information on groups of concordance classes of PL self-knottings from these decompositions and a number of fairly explicitly constructed examples of self-knottings. I would like to thank by thesis advisor R. J. Milgram for many helpful discussions.

I. Sullivan's classification of PL homotopy $\widetilde{C P}^{n}$ proceeds as follows: Given a homotopy equivalence $h: \widetilde{C P}^{n} \rightarrow C P^{n}$ make $h$ transverse regular to $C P^{J} \subset \widetilde{C P^{n}}$, the standard inclusion. The restriction of $h$ to the transverse inverse image $h^{-1}\left(C P^{j}\right)=N^{2 j} \subset \widetilde{C P^{n}}$ is a degree one normal map 
with simply connected surgery obstruction

$$
\sigma_{j} \in P_{2 J}=\left\{\begin{array}{ll}
Z, & j \text { even } \\
Z / 2 Z, & j \text { odd }
\end{array}\right\} .
$$

For $j=2, \ldots, n-1$ these obstruction invariants yield a complete enumeration - i.e. the set of PL isomorphism classes of $\widetilde{C P}^{n}$ is set-isomorphic to the product $Z \times Z_{2} \times Z \times \cdots \times P_{2(n-1)}$ with $n-2$ factors.

We will use the following notation to specify elements with this classification:

$$
\widetilde{C P^{n}} \leftrightarrow\left(\sigma_{2}, \sigma_{3}, \ldots, \sigma_{n-1}\right)
$$

will denote the PL homotopy $\widetilde{C P}^{n}$ with invariants $\sigma_{j} \in P_{2 j}$ in Sullivan's enumeration.

We recall that a PL homeomorphism $f: M \rightarrow M$ is a "self-knotting" and $M$ is said to be "self knotted" if $f$ is homotopic but not PL isotopic to the identity. Also, PL homeomorphisms $f, g: M \rightarrow M$ are "PL concordant" (pseudo-isotopic) if we have a PL homeomorphism $F: M \times I \rightarrow M \times I$ with $F(m, 0)=(f(m), 0)$ and $F(m, 1)=(g(m), 1)$ for $m \in M$. We then define:

(2) $S K(M)=$ "the group (under composition of maps) of PL concordance classes of PL self-knottings of $M . "$

Unless otherwise noted " $C P^{j} \subset C P^{n}$ " means the standard embedding of $C P^{j}$ onto the first $(j+1)$ homogeneous coordinates of $C P^{n}$ or a smooth ambient isotope of this embedding. In this context we define:

(3) $\nu_{N}\left(C P^{j}\right)=$ "the smooth tubular disc bundle neighborhood of the embedding $C P^{j} \subset C P^{N}$."

Our results are as follows:

THeOREM A. For $n \geq 4$ and $\sigma_{2} \equiv 0$ (2) every $\widetilde{C P^{n}} \leftrightarrow\left(\sigma_{2}, \sigma_{3}, \ldots, \sigma_{n-1}\right)$ is $P L$ homeomorphic to the identification space

$$
\left[\widehat{C P}^{n}-\nu_{n}\left(C P^{1}\right)\right] \cup_{\varphi_{\sigma_{n-1}}}\left[\nu_{n}\left(C P^{1}\right)\right]
$$

where $\widehat{C P}^{n} \leftrightarrow\left(\sigma_{2}, \sigma_{3}, \ldots, \sigma_{n-2}, 0\right)$ and the identification is over a PL homeomorphism

$$
\varphi_{\sigma_{n-1}}: \partial \nu_{n}\left(C P^{1}\right) \rightarrow \partial \nu_{n}\left(C P^{1}\right)
$$

We prove Theorem A in Part II by a careful description of Sullivan's classification and an easy $h$-cobordism argument. 
An immediate consequence of Theorem $\mathrm{A}$ is the decomposition of $\widetilde{C P}^{n+1} \leftrightarrow\left(0, \ldots, 0, \sigma_{n}\right)$ into

$$
\widetilde{C P}^{n+1}=\left[C P^{n+1}-\nu\left(C P^{1}\right)\right] \cup_{\varphi_{0}}\left[\nu\left(C P^{1}\right)\right] .
$$

THEOREM B. For every $n \geq 4$ and non-zero $\tau \in P_{2 n}$ there is a $P L$ self-knotting

$$
\varphi_{\tau}: \partial \nu_{n+1}\left(C P^{1}\right) \rightarrow \partial \nu_{n+1}\left(C P^{1}\right)
$$

which will suffice for the glueing homeomorphism in Theorem A.

We establish this theorem by an explicit construction of $\varphi_{\tau}$ in Part III.

II. Here we prove Theorem $\mathrm{A}$ by beginning with a construction which shows how to obtain $\widetilde{C P}^{n+1} \leftrightarrow\left(\sigma_{2}, \ldots, \sigma_{n-1}, \sigma_{n}\right)$ from $\widetilde{C P}^{n} \leftrightarrow$ $\left(\sigma_{2}, \ldots, \sigma_{n-1}\right)$ for $n \geq 4$ :

Let $h: \widetilde{C P^{n}} \rightarrow C P^{n}$ be a homotopy equivalence, and let $M^{2 n}$ be the compact $(n-1)$-connected Milnor or Kervaire manifold of Index $8 \sigma_{n}$ or Kervaire-Arf invariant $\sigma_{n}$ as the case may be [4]. Let $r: M^{2 n} \rightarrow \mathrm{S}^{2 n}$ be a degree one map. Then $h \# r: \widetilde{C P^{n}} \# M^{2 n} \rightarrow C P^{n} \# S^{2 n}=C P^{n}$ is a degree one normal map with 1-connected surgery obstruction $\sigma_{n}$. We define $\hat{H}$ as the $D^{2}$ bundle over $\widetilde{C P^{n}} \# M^{2 n}$ induced by $h \# r$ from $H$, the disc bundle associated to the complex line bundle over $C P^{n}$. Let $\hat{h}: \hat{H} \rightarrow H$ be the bundle mapping. We note that the map $h \# r$ is $(n-1)$-connected with homological kernel $K_{n}=\pi_{n}\left(M_{0}^{2 n}\right)$ where $M_{0}^{2 n}=M^{2 n}-D^{2 n}$. The bundle $\hat{H}$ is trivial over $M_{0}^{2 n}$ since $M_{0}^{2 n}=(h \# r)^{-1}$ (point). In $M_{0}^{2 n} \times D^{2}$ we can represent $\pi_{n}\left(M_{0}^{2 n}\right)$ by disjointly embedded spheres $S^{n} \hookrightarrow M_{0}^{2 n} \times S^{1}$ with trivial normal bundles. This follows by general position and the fact that the normal bundles of the generating spheres $S^{n} \subset M_{0}^{2 n}$ are the stably trivial tangent disc bundles $\tau\left(S^{n}\right)$. We now attach a solid handle $D^{n+1} \times$ $D^{n+1}$ along $S^{n} \times D^{n+1} \subset M_{0}^{2 n} \times S^{1}$ for each generator of $\pi_{n}\left(M_{0}^{2 n}\right)$ and extend the map $\hat{h}$ across these bundles. This is possible since the embedded spheres are in the homotopy kernel of $\hat{h}$. Call the resulting PL manifold $\tilde{H}$ and the extended map $\hat{h}: \hat{H} \rightarrow H$. In the process of extending $\hat{h}$ across the handles, we may guarantee that $\tilde{h}$ is a map of pairs $(\tilde{H}, \partial) \rightarrow$ $(H, \partial)$. We observe, then, the:

Proposition. $\tilde{h}:(\tilde{H}, \partial) \rightarrow(H, \partial)$ is a homotopy equivalence of pairs. 
This follows directly from the construction as $\tilde{H}$ deformation retracts onto $\widetilde{C P^{n}} \# M^{2 n} \cup\left\{e_{\alpha}^{n}\right\}$ where the $n$-cells $e_{\alpha}^{n}$ are attached so as to kill the entire homology kernel of $(h \# r)$. Hence $\tilde{h}: \tilde{H} \rightarrow H$ is a homology isomorphism, and as $\tilde{H}$ is 1-connected we have by Whitehead's theorem that it is a homotopy equivalence. The restriction of $\tilde{h}$ to the boundary is likewise a homology isomorphism as the boundaries, $D^{n+1} \times S_{\alpha}^{n}$, of the solid handles are precisely the surgeries needed to cobord $\hat{h}: \partial \hat{H} \rightarrow \partial H$ to a homotopy equivalence.

In particular as $n \geq 3$ we note that the boundary manifold, $\partial \tilde{H}$, is a PL $(2 n+1)$-sphere by the Poincaré conjecture. Thus, we attach $D^{2 n+2}$ to $\tilde{H}$ as the PL cone on $\partial \tilde{H}$ and define:

$$
\widetilde{C P}^{n+1}=\tilde{H} \cup c(\partial \tilde{H}) \text { and } h: \widetilde{C P}^{n+1} \rightarrow C P^{n+1}=H \cup c(\partial H)
$$

by radial extension of $\tilde{h}$ into $c(\partial \tilde{H})$.

Observe that $h$ has "built-in" transverse inverse image $\widetilde{C P^{n}} \# M^{2 n}=$ $h^{-1}\left(C P^{n}\right)$ with surgery obstruction $\sigma_{n}$. Hence, this $\widetilde{C P}^{n+1} \leftrightarrow\left(\sigma_{2}, \ldots\right.$, $\left.\sigma_{n-1}, \sigma_{n}\right)$ is the space we require.

Now, given $\widetilde{C P}^{n} \leftrightarrow\left(\sigma_{2}, \ldots, \sigma_{n-1}\right)$ let us consider a bit more closely the suspension and generalized suspension constructions described above. First, assume the homotopy equivalence

$$
h: \widetilde{C P}^{n} \rightarrow C P^{n}
$$

is the identity map on a disc $D^{2 n} \subset \widetilde{C P^{n}}$. Let $\widetilde{C P}_{0}^{n}=\widetilde{C P}^{n}-D^{2 n}, M_{0}^{2 n}=$ $M^{2 n}-D^{2 n}$ and observe that $\widetilde{C P}^{n} \# M^{2 n}=\widetilde{C P}_{0}^{n} \cup_{\partial} M_{0}^{2 n}$. Now, let $\widetilde{C P}^{n+1}$ $\leftrightarrow\left(\sigma_{2}, \ldots, \sigma_{n-1}, 0\right)$ be the suspension ${ }^{1}$ of $\widetilde{C P}^{n}$ with homotopy equivalence

$$
\tilde{h}: \widetilde{C P}^{n+1} \rightarrow C P^{n+1}
$$

and $\widehat{C P}^{n} \leftrightarrow\left(\sigma_{2}, \ldots, \sigma_{n-1}, \sigma_{n}\right)$ be the general suspension of $\widetilde{C P}^{n}$ with homotopy equivalence

$$
\hat{h}: \widehat{C P}^{n+1} \rightarrow C P^{n+1} \text {. }
$$

Let $D^{2 n} \subset C P^{n}$ be the image $h\left(D^{2 n}\right)$ and let $C P^{1}=S^{2} \subset C P^{n+1}$ be represented as $D_{*}^{2} \cup c\left(\partial D_{*}^{2}\right)$ in $C P^{n+1}=H \cup c(\partial H)$ with $D_{*}^{2}$ the fiber in $H$ over the center of the disc $D^{2 n}$. Then $\nu_{n+1}\left(C P^{1}\right) \subset C P^{n+1}$ may be represented as the set $D_{*}^{2} \times D^{2 n} \cup c(\partial H)$, a $D^{2 n}$ bundle over the sphere $S^{2}=D_{*}^{2} \cup c\left(\partial D_{*}^{2}\right)$.

Now let $\tilde{V}=\tilde{h}^{-1}\left(\nu_{n+1}\left(C P^{1}\right)\right)$ and $\hat{V}=\hat{h}^{-1}\left(\nu_{n+1}\left(C P^{1}\right)\right)$ in $\widetilde{C P}^{n+1}$ and $\widehat{C P}^{n+1}$ respectively. We observe directly from the constructions that

\footnotetext{
${ }^{1}$ We say $\widetilde{C P^{n+1}} \leftrightarrow\left(\sigma_{2}, \sigma_{3}, \ldots, \sigma_{n-1}, 0\right)$ in the "suspension" of $\widetilde{C P^{n}} \leftrightarrow\left(\sigma_{2}, \sigma_{3}, \ldots, \sigma_{n-1}\right)$ as it is precisely the Thom complex of the line bundle induced over $\widetilde{C P}^{n}$.
} 
$\widetilde{C P}^{n+1}-\tilde{V}$ and $\widehat{C P}^{n+1}-\hat{V}$ are precisely the same spaces. To prove Theorem A we must show that $\tilde{V}$ and $\hat{V}$ are PL homeomorphic to $\nu_{n+1}\left(C P^{1}\right)$.

LEMMA 1. $\tilde{V} \cong \nu_{n+1}\left(C P^{1}\right)$ if $\sigma_{2}$ is even.

We observe this from PL block bundle theory as follows: by construction $\tilde{V}$ is the union of two discs $D_{*}^{2} \times D^{2 n}$ and $c(\partial \tilde{H})=D^{2 n+2}$ along $S_{*}^{1} \times D^{2 n}$. Hence $\tilde{V}$ is trivially a block bundle regular neighborhood of $C P^{1}=D_{*}^{2} \cup c\left(\partial D_{*}^{2}\right)$. Assume the obstruction $\sigma_{2}$ is even. Then as noted by Sullivan ([23] p. 43) the splitting obstruction of the homotopy equivalence

$$
\tilde{h}: \widetilde{C P}^{n+1} \rightarrow C P^{n+1}
$$

along $C P^{1}$ vanishes as it is the $\bmod 2$ reduction of $\sigma_{2}$. Hence, by a homotopic deformation we may conclude that the transverse inverse image of $C P^{1}$ by $\tilde{h}$ is $C P^{1} \subset \widetilde{C P^{n+1}}$. Moreover, as any two homotopic PL embeddings of $C P^{1} \subset \widetilde{C P}^{n+1}$ are ambiently PL isotopic (for $n \geq 2$ by Cor. 5.9 p. 65 [21]), we see by appeal to the uniqueness of normal block bundles (regular neighborhoods) [20] that $\tilde{V}$ is block bundle isomorphic to the bundle induced from $\nu_{n+1}\left(C P^{1}\right)$ by $\tilde{h}$. Conversely, the same argument on the homotopy inverse of $\tilde{h}$ implies $\nu_{n+1}\left(C P^{\mathrm{l}}\right)$ is block bundle induced from $\tilde{V}$. As we are in the stable block and vector bundle range and $\pi_{2} B_{\mathrm{PL}}=\pi_{2} B_{0}=Z_{2}$ we can conclude that $\tilde{C}$ and $\nu\left(C P^{1}\right)$ are block bundle isomorphic; hence PL homeomorphic.

\section{LEMMA 2. $\hat{V} \simeq S^{2}$ (homotopy equivalent).}

Proof. By construction $\hat{V}=D^{2} \times M_{0}^{2 n} \cup X \cup c(\partial H)$ where $X$ represents the solid handles we attached along $S^{1} \times M_{0}^{2 n}$ to kill the homology kernel of $\hat{h}$. The manifold $D^{2} \times M_{0}^{2 n} \cup X$ is simply-connected with simply connected boundary and the homology of a point; hence by Smale's theorem (Thm. 1.1 [22]) it is a PL disc $D^{2 n+2}$. Thus, $\hat{V}=D^{2 n+2} \cup_{W} D^{2 n+2}$ where $W$ is the complement of the embedding

$$
D^{2} \times S^{2 n-1} \subset S^{2 n+1}=\partial D^{2 n+2}
$$

and $S^{2 n-1}=\partial M_{0}^{2 n}$. By the Mayer-Vietoris sequence we know that $W$ is a homology circle. Then, by a second application of the Mayer-Vietoris sequence to the union $D^{2 n+2} \cup_{W} D^{2 n+2}$ we see that $\hat{V}$ is a homology $S^{2}$. Finally, by the Van Kampen theorem $\hat{V}$ is 1 -connected and we apply the Whitehead theorem for CW complexes. 
LEMMA 3. $\hat{V} \cong \nu_{n+1}\left(C P^{1}\right)$.

Proof. $\partial \hat{V}=\partial\left[C P^{n+1}-\hat{V}\right]=\partial\left[C P^{n+1}-\tilde{V}\right]=\partial \tilde{V} \cong \partial v_{n+1}\left(C P^{1}\right)$ by Lemma 1. Let $S^{2} \subset \hat{V}$ be a homotopy equivalence and a PL embedding via Whitney's embedding theorem. Then $S^{2} \subset \hat{V} \subset \widehat{C P}^{n+1}$ is homotopic to the standard embedding $C P^{1} \subset \widehat{C P}^{n+1}$, and as before, the PL block bundle neighborhoods of these two embeddings must be isomorphic. Let $\nu \subset \hat{V}$ be this block bundle. We note that

$$
\partial \nu=\partial \nu_{n+1}\left(C P^{1}\right) \cong \partial \tilde{V}=\partial \hat{V}
$$

by the previous lemmas. Hence, if

$$
\hat{V}-\nu=Y
$$

we have $\partial Y=\partial \hat{V} \cup . \partial \nu$, two copies of the same manifold.

We consider the Mayer-Vietoris sequence for the union $\hat{V}=Y \cup \nu$ over $\partial \nu=Y \cap \nu$ :

$$
\cdots \rightarrow H_{1}(\partial \nu) \stackrel{i_{1_{*}}-i_{2 *}}{\rightarrow} H_{1}(\nu) \oplus H_{q}(Y) \stackrel{j_{1 *}-j_{2 *}}{\rightarrow} H_{1}(\hat{V}) \rightarrow \cdots
$$

where

$$
\begin{aligned}
& i_{1}: \partial \nu \hookrightarrow \nu, \quad j_{1}: \nu \hookrightarrow \hat{V}, \\
& i_{2}: \partial \nu \hookrightarrow Y, \quad j_{2}: Y \hookrightarrow \hat{V} .
\end{aligned}
$$

Since $\nu$ and $V$ are homotopy 2 -spheres and $j_{1}$ is a homotopy equivalence, we see that for $q \neq 2, i_{2_{*}}: H_{q}(\partial \nu) \rightarrow H_{q}(Y)$ must be an isomorphism. When $q=2$ the sequence becomes:

$$
Z \stackrel{1-i_{2 *}}{\rightarrow} Z \oplus A \stackrel{1+j_{2 *}}{\rightarrow} Z, \quad A=H_{2}(Y)
$$

from which we obtain $i_{2_{*}}$ are isomorphisms $Z \stackrel{i_{2 *}}{\rightarrow} A \stackrel{j_{2 *}}{\rightarrow} Z$. Thus, $i_{2}: \partial \nu \subset Y$ is a homology isomorphism, and in fact, a homotopy equivalence since $\hat{V}=Y \cup \nu$ and $\hat{V}, \nu, \partial \nu$ are all 1-connected so that by Van Kampen's theorem $Y$ is 1-connected.

We show next that $\partial \hat{V} \stackrel{i}{\subset} Y$ is a homology isomorphism so that $Y$ is a $h$-cobordism from $\partial \nu$ to $\partial \hat{V}$-i.e. $Y \cong \partial \nu \times I$ and $\hat{V}=Y \cup \nu \cong \nu \cong$ $\tilde{V} \nu_{n+1}\left(C P^{1}\right)$ as required. 
We know already that $\partial \hat{V} \simeq Y$ as $\partial \hat{V} \cong \partial \nu \simeq Y$. Moreover, $\partial \nu \cong$ $\partial \nu_{n+1}\left(C P^{1}\right)$ is an $S^{2 n-1}$ bundle over $S^{2}$. Hence, by the Serre Spectral Sequence we have

$$
H_{p}(Y)=H_{p}(\partial \hat{V})= \begin{cases}Z & \text { if } p=0,2,2 n-1,2 n+1 \\ 0 & \text { otherwise }\end{cases}
$$

Then, the exact sequence of the pair $(\hat{V}, \partial \hat{V})$ is:

$$
0=H_{3}(\hat{V}, \partial \hat{V}) \rightarrow \underset{\|}{H_{2}(\partial \hat{V})} \rightarrow \underset{H_{2}(\hat{V}) \rightarrow H_{1}(\hat{V}, \partial \hat{V})=0}{\|}
$$

where the first and last groups are 0 by Poincare Duality. Thus, the inclusion $\partial \hat{V} \subset Y \subset \hat{V}$ is a homology isomorphism through $p=2$.

Now, consider the composition $f: \partial \hat{V} \stackrel{i}{\hookrightarrow} \mathrm{Y} \rightarrow \partial \hat{V}$ where the second map is a homotopy equivalence. Then $f_{*}: H_{p}(\partial \hat{V}) \rightarrow H_{p}(\partial \hat{V})$ is an isomorphism for $p \leq 2$, and by Poincare Duality so is $f^{*}: H^{I}(\partial \hat{V}) \rightarrow H^{I}(\partial \hat{V})$ for $q=2 n-1,2 n, 2 n+1$. By the Universal Coefficient Theorem $f_{*}$ is an isomorphism for $p=2 n-1,2 n, 2 n+1$ and so for all $p$. Thus, $f$ is a homotopy equivalence, and so is $i$.

Theorem $\mathrm{A}$ is now an immediate consequence of the last lemma as we have:

$$
\begin{gathered}
\widehat{C P}^{n+1} \leftrightarrow\left(\sigma_{2}, \ldots, \sigma_{n-1}, \sigma_{n}\right)=\left[C P^{n+1}-\tilde{V}\right] \cup \hat{V} \\
\widetilde{C P}^{n} \leftrightarrow\left(\sigma_{2}, \ldots, \sigma_{n-1}, 0\right)=\left[C P^{n+1}-\nu_{n+1}\left(C P^{1}\right)\right] \cup_{\alpha_{\sigma_{n}}} \nu_{n+1}\left(C P^{1}\right)
\end{gathered}
$$

where we have identified $\tilde{V}$ with $\nu_{n+1}\left(C P^{1}\right)$ by Lemma 1 , and the PL homeomorphism

$$
\varphi_{\sigma_{n}}: \partial\left[\widetilde{C P}^{n+1}-\nu\left(C P^{1}\right)\right] \rightarrow \partial \nu\left(C P^{1}\right)
$$

comes from the restriction to the boundary of the PL homeomorphism $\hat{V} \rightarrow \nu_{n+1}\left(C P^{1}\right)$ of Lemma 3.

III. Construction of the self-knotting $\varphi_{\sigma}$ : Here we construct for $n \geq 4$ a PL self-knotting

$$
\varphi_{\sigma}: \partial \nu_{n+1}\left(C P^{1}\right) \rightarrow \partial \nu_{n+1}\left(C P^{1}\right)
$$

with the property that it extends to a homotopy equivalence

$$
\bar{\varphi}_{\sigma}: \nu_{n+1}\left(C P^{1}\right) \rightarrow \nu_{n+1}\left(C P^{1}\right)
$$


which has a transverse-inverse image

$$
M_{0}^{2 n}=\bar{\varphi}_{\sigma}^{-1}\left(D^{2 n}\right)
$$

on a fiber $D^{2 n}$. Clearly such a $\varphi_{\sigma}$ will suffice for the map in Theorem A.

We begin the construction by defining

$$
\Sigma_{\sigma}^{2 n-1} \subset S^{2 n+1}
$$

to be the smooth Brieskorn knot represented as the link of the singularity on the hypersurface in $C^{n+1}$ defined by

$$
p(Z)=\left\{\begin{array}{l}
Z_{0}^{6 \sigma-1}+Z_{1}^{3}+Z_{2}^{2}+\cdots+Z_{n}^{2}, \quad n \text { even, } \\
Z_{0}^{3}+Z_{1}^{2}+\cdots+Z_{n}^{2}, \quad n \text { odd. }
\end{array}\right.
$$

It is well-known that $S^{2 n+1}-\Sigma_{\sigma}^{2 n-1}$ is a smooth fiber bundle over the circle with fiber $M_{0}^{2 n}$, the smooth Milnor or Kervaire manifold with surgery invariant $\sigma$.

Now, let $S^{1} \subset S^{2 n+1}$ be a fiber on the boundary of the smooth tubular neighborhood $D^{2} \times \Sigma_{\sigma}^{2 n-1}$ of the knot (a trivial bundle as $\pi_{2 n-1}(\operatorname{SO}(2))=0$ for $\left.n>1\right)$. Since $n>1$ this circle $S^{1}$ is smoothly unknotted in $S^{2 n+1}$ so that the complement of a small tube $S^{1} \times D^{2 n}$ about it is diffeomorphic to $D^{2} \times S^{2 n-1}$. Hence the knot $\Sigma_{\sigma}^{2 n-1}$ lies in this complement with a trivial normal bundle and we can therefore define:

$$
\beta: D^{2} \times \Sigma_{\sigma}^{2 n-1} \hookrightarrow D^{2} \times S^{2 n-1}
$$

as this embedding. Let $W^{2 n+1}$ be the complement of this smooth embedding. Then we observe:

(a) $\partial W=S^{1} \times S^{2 n-1} \cup S^{1} \times \Sigma_{\sigma}^{2 n-1}$.

(b) $W$ is a smooth fiber bundle over the circle $S^{1}$ with fiber $F^{2 n}=$ $M_{0}^{2 n}-D^{2}$ and $\partial F=S^{2 n-1} \cup \Sigma_{\sigma}^{2 n-1}$.

(c) the bundle projection is trivial on $\partial W \rightarrow S^{1}$.

Now, using the smooth embedding $\beta$ we define a piecewise-linear embedding

$$
\gamma_{\sigma}: D^{2} \times S^{2 n-1} \hookrightarrow D^{2} \times S^{2 n-1}
$$

as the composite map

$$
D^{2} \times S^{2 n-1} \stackrel{\text { id } \times \alpha_{\sigma}}{\rightarrow} D^{2} \times \Sigma_{\sigma}^{2 n-1} \stackrel{\beta}{\rightarrow} D^{2} \times S^{2 n-1}
$$

where $\alpha_{\sigma}: S^{2 n-1} \rightarrow \Sigma_{\sigma}^{2 n-1}$ is a specific PL homeomorphism. 
We now describe the normal bundle $\nu_{n+1}\left(C P^{1}\right)$ in $C P^{n+1}$ as:

$$
\nu_{n+1}\left(C P^{1}\right)=D_{-}^{2} \times S^{2 n-1} \cup_{\rho} D_{+}^{2} \times S^{2 n-1}
$$

(*) where $\rho: S^{1} \times S^{2 n-1} \rightarrow S^{1} \times S^{2 n-1}$ is a smooth bundle automorphism representing an element in $\pi_{1}(\operatorname{SO}(2 n))=Z / 2 Z(n>1)$. [We note in fact that $\gamma_{n+1}\left(C P^{1}\right)$ is trivial for $n$ even and non-trivial for $n$ odd as it is the Whitney sum of $n$ copies of the canonical line bundle over $C P^{1}=S^{2}$.]

In the above description we are expressing $C P^{1}$ as $S^{2}=D_{-}^{2} \cup D_{+}^{2}$. Using this representation we will define the self-knotting $\varphi_{\sigma}$ by showing that the PL embedding

$$
\gamma_{\sigma}: D_{+}^{2} \times S^{2 n-1} \hookrightarrow D_{+}^{2} \times S^{2 n-1}
$$

may be extended to a PL homeomorphism on all of $V_{n+1}\left(C P^{1}\right)$. We will show this using the very agreeable bundle structure on the complement $W$ of the embedding $\gamma_{\sigma}$.

The map

$$
\varphi_{\sigma}: D_{-}^{2} \times S^{2 n-1} \cup_{\rho} D_{+}^{2} \times S^{2 n-1} \rightarrow D_{-}^{2} \times S^{2 n-1} \cup_{\rho} D_{+}^{2} \times S^{2 n-1}
$$

will in fact be defined as the union of three maps -

$$
\begin{gathered}
\gamma_{\sigma}: D_{+}^{2} \times S^{2 n-1} \hookrightarrow D_{+}^{2} \times S^{2 n-1}, \\
\eta: \tilde{W}^{2 n+1} \rightarrow W^{2 n+1}, \\
\text { id } \times \mu: D^{2} \times \Sigma_{-\sigma}^{2 n-1} \rightarrow D_{-}^{2} \times S^{2 n-1}
\end{gathered}
$$

where $\eta$ is a bundle homeomorphism of bundles over $S^{1}$ and $\mu: \Sigma_{-\sigma}^{2 n-1} \rightarrow$ $S^{2 n-1}$ is a PL homeomorphism and

$$
D^{2} \times \Sigma_{-\sigma}^{2 n-1} \cup \tilde{W}^{2 n+1}=D_{-}^{2} \times S^{2 n+1} .
$$

Essentially what we are producing in this construction is a map with the symmetric property that $\varphi_{\sigma}$ embeds a fiber (the core of $D_{+}^{2} \times S^{2 n-1}$ ) piecewise linearly onto the smooth fibered knot $\Sigma_{-\sigma}^{2 n-1} \subset D_{-}^{2} \times S^{2 n-1}$ while $\varphi_{\sigma}^{-1}$ embeds a fiber (the core of $D_{-}^{2} \times S^{2 n-1}$ ) piecewise linearly onto the smooth fibered knot $\Sigma_{\sigma}^{2 n-1} \subset D_{-}^{2} \times S^{2 n-1}$.

The construction will be completed by (a) defining the bundle $\tilde{W}$ and the bundle map $\eta$ in (2), (b) showing that $D^{2} \times \Sigma_{-\sigma}^{2 n-1} \cup \tilde{W}$ is in fact $D^{2} \times S^{2 n-1}$ by a PL homeomorphism which is the identity on the boundary, (c) showing that the maps (1), (2), (3) agree on boundaries after taking the defining automorphism $\rho$ into account, and finally by (d) showing that $\varphi_{\sigma}$ is homotopic to the identity. 
We define the bundle $\tilde{W}$ over $S^{1}$ by defining its fiber $\tilde{F}$ and its monodromy map $\tilde{h}: \tilde{F} \rightarrow \tilde{F}$.

Recall that the $2 n$-manifold $F$ (fiber of $W$ ) is $(n-1)$ connected and that $\partial F=S^{2 n-1} \cup \Sigma_{-\sigma}^{2 n-}$ where the smooth exotic sphere is defined as $\Sigma_{\sigma}^{2 n-1}=D_{-}^{2 n-1} \cup_{\sigma}^{\cdot} D_{+}^{2 n+1}$ and $\sigma: S^{2 n-2} \rightarrow S^{2 n-2}$ is an exotic diffeomorphism.

Let $I \subset F$ be a path connecting the centers of the discs $D_{+}^{2 n-1}$ and $D_{+}^{2 n-1}$ of $\Sigma_{\sigma}^{2 n-1}$ and $S^{2 n-1}$. Then a tubular neighborhood of $I$ is $I \times D_{+}^{2 n-1}$. We define $\tilde{F}$ as the smooth manifold

$$
\tilde{F}=\left[F-I \times D_{+}^{2 n-1}\right] \cup\left[I \times D_{+}^{2 n-1}\right]
$$

where the union is taken over the diffeomorphism

$$
\mathrm{id}_{I} \times \sigma^{-1}: I \times S^{2 n-2} \rightarrow I \times S^{2 n-2} .
$$

Then $\partial \tilde{F}=\Sigma_{-\sigma}^{2 n-} \cup S^{2 n-1}$ as a smooth manifold and we can define a PL homeomorphism

$$
\hat{\eta}: \tilde{F} \rightarrow F
$$

where $\hat{\eta}$ is the identity on $F-I \times D_{+}^{2 n-1}$ and is id $\mathrm{id}_{I} \times$ (cone extension of $\sigma)$ on $I \times D_{+}^{2 n-1}$.

Then we define the monodromy $\tilde{h}: \tilde{F} \rightarrow \tilde{F}$ as the composite map

$$
\tilde{h}=\hat{\eta}^{-1} \circ h \circ \hat{\eta}
$$

where $h: F \rightarrow F$ is the monodromy map defining the bundle $W$. Since $\partial W$ is a trivial bundle we know that $h$ is the identiy map on $\partial F$. Hence, $\tilde{h}$ is the identity on $\partial \tilde{F}$ and the bundle $\tilde{W}$ has the trivial boundary

$$
\partial \tilde{W}=S^{1} \times \Sigma_{-\sigma}^{2 n-} \cup S^{1} \times S^{2 n-1} .
$$

Since $\hat{\eta} \circ \tilde{h}=h \circ \hat{\eta}$ the PL homeomorphism $\hat{\eta}: \tilde{F} \rightarrow F$ induces a well-defined bundle homeomorphism

$$
\eta: \tilde{W}^{2 n+1} \rightarrow W^{2 n+1} .
$$

Restricted to the boundary $\eta$ is a pair of bundle maps

$$
\begin{gathered}
\operatorname{id}_{S^{1}} \times \alpha_{-\sigma}^{-1}: S^{1} \times \Sigma_{-\sigma}^{2 n-} \rightarrow S^{1} \times S^{2 n-1}, \\
\operatorname{id}_{S^{1}} \times \alpha_{\sigma}: S^{1} \times S^{2 n-1} \rightarrow S^{1} \times \Sigma_{\sigma}^{2 n-1}
\end{gathered}
$$

where the PL homeomorphism $\alpha_{-\sigma}$ and $\alpha_{\sigma}$ are the identity on $D_{-}^{2 n-1}$ and the cone extension of $\sigma^{-1}$ and $\sigma$ respectively on $D_{+}^{2 n-1}$. 
We next embed $\tilde{W}$ in $D^{2} \times S^{2 n-1}$ as a knot complement which will act as an inverse to $W$ :

Recall the bundle isomorphism

$$
\rho: S^{1} \times S^{2 n-1} \rightarrow S^{1} \times S^{2 n-1}
$$

which defines $\partial \nu_{n+1}\left(C P^{1}\right)$. We define a PL bundle map

$$
\hat{\rho}: S^{1} \times \Sigma_{-\sigma}^{2 n-1} \rightarrow S^{1} \times \Sigma_{-\sigma}^{2 n-1}
$$

as the composite: $\hat{\rho}=\left(\mathrm{id}_{S^{1}} \times \alpha_{-\sigma}\right) \cdot \rho \cdot\left(\operatorname{id}_{S^{1}} \times \alpha_{-\sigma}\right)^{-1}$. We consider the PL manifold

$$
D^{2} \times \Sigma_{-\sigma}^{2 n-1} \cup_{\hat{\rho}} \tilde{W}^{2 n+1}
$$

where the union is over the appropriate component of $\partial \tilde{W}$ and show:

Proposition. The PL manifold $D^{2} \times \Sigma_{-\sigma}^{2 n-1} \cup_{\hat{\rho}} \tilde{W}^{2 n+1}$ is isomorphic to $D^{2} \times S^{2 n-1}$ by a PL homeomorphism $\Lambda$ which restricted to the boundary $S^{1} \times S^{2 n-1}$ is an $S^{2 n-1}$ bundle isomorphism $\lambda$.

Proof. We recall from the definition of $W^{2 n+1}$ that $S^{1} \times D^{2 n} \cup W^{2 n+1}$ is the knot complement of our original Brieskorn knot and so has the homology of $S^{1}$. A simple exercise with the Mayer-Vietoris sequence implies then that the manifold $\tilde{W}^{2 n+1} \cup S^{1} \times D^{2 n}$ likewise is a homology circle, and a second application of the sequence implies that the PL manifold.

$$
P^{2 n+1}=D^{2} \times \Sigma_{-\sigma}^{2 n-1} \cup_{\hat{\rho}} \tilde{W} \cup S^{1} \times D^{2 n}
$$

has the homology of $S^{2 n+1}$. Moreover, $P^{2 n+1}$ is simply connected since $\hat{W} \cup S^{1} \times D^{2 n}$ fibers over $S^{1}$ with fiber $\tilde{F}^{2 n} \cup D^{2 n}$ which is $(n-1)$ connected. Hence $\pi_{1}\left(\tilde{W} \cup S^{1} \times D^{2 n}\right)=Z$ and by the Van Kampen theorem on the union

$$
\left[D^{2} \times \Sigma_{-\sigma}^{2 n-1}\right] \cup_{S^{1} \times \Sigma_{-\sigma}}\left[\tilde{W} \cup S^{1} \times D^{2 n}\right]
$$

we have $\pi_{1}\left(P^{2 n+1}\right)=0$. By the Hurewicz and Whitehead theorems any simply-connected homology sphere is a homotopy sphere, and by the generalized Poincaré conjecture $(2 n+1 \geq 9) P^{2 n+1}$ is a PL sphere.

The identification $D^{2} \times \Sigma_{-\sigma}^{2 n-1} \cup \tilde{W} \mathrm{~S}^{1} \times D^{2 n} \cong S^{2 n+1}$ provides a PL embedding $S^{1} \stackrel{i}{\subset} S^{2 n+1}$ and exhibits $i\left(S^{1} \times D^{2 n}\right) \subset S^{2 n+1}$ as a representative for the PL normal microbundle to this embedding. We apply a 
theorem due to Lashof and Rothenberg (Thm. 7.3 in [13]) to obtain a piecewise differentiable homeomorphism $g: S^{2 n+1} \rightarrow S^{2 n+1}$ so that $g \circ i$ : $S^{1} \times D^{2 n} \rightarrow S^{2 n+1}$ is the smooth vector bundle to the smooth embedding $g \circ i: S^{1} \rightarrow S^{2 n+1}$. By smoothly unknotting this circle and applying the smooth tubular neighborhood theorem we obtain a diffeomorphism $h$ : $S^{2 n+1} \rightarrow S^{2 n+1}$ so that

$$
\begin{array}{ccc}
h \circ g \circ i: S^{1} \times D^{2 n} & \rightarrow & S^{2 n+1} \\
& \bar{\lambda} \searrow & \uparrow j \\
& & S^{1} \times D^{2 n}
\end{array}
$$

commutes where $j$ is the standard embedding and $\bar{\lambda}$ is a vector bundle isomorphism. Hence, the restriction map

$$
\begin{array}{r}
h \circ g \mid: S^{2 n+1}-i\left(S^{1} \times D^{2 n}\right) \rightarrow S^{2 n+1}-j\left(S^{1} \times D^{2 n}\right) \\
\| \\
D^{2} \times S^{2 n-1}
\end{array}
$$

defines a piecewise differentiable homeomorphism

$$
\Lambda:\left[D^{2} \times \Sigma_{-\sigma}^{2 n-} \cup_{\hat{\rho}} \hat{W}\right] \rightarrow D^{2} \times S^{2 n-1}
$$

which restricts as $\lambda=\bar{\lambda}$ on the boundary. Finally, we observe that (cf. Cor. 10.13 in [19]) we may choose a smooth triangulation of $D^{2} \times S^{2 n-1}$ so that $\Lambda$ is PL. Now, using the homeomorphisms $\Lambda$ and $\eta$ we define a PL homeomorphism:

$$
\varphi_{\sigma}: \xi \rightarrow \partial \nu_{n+1}\left(C P^{1}\right)
$$

where $\xi$ is the $S^{2 n-1}$ bundle over $C P^{1}=S^{2}$ defined by $\lambda^{-1}$ :

$$
\begin{gathered}
\xi=D_{-}^{2} \times S^{2 n-1} \cup_{\lambda^{-1}} D_{+}^{2} \times S^{2 n-1} \\
\stackrel{\Lambda^{-1} \cup \mathrm{id}}{\rightarrow} D^{2} \times \Sigma_{-\sigma}^{2 n-1} \cup_{\hat{\rho}} \tilde{W}^{2 n+1} \cup_{\mathrm{id}} D_{+}^{2} \times S^{2 n-1} \\
\stackrel{\left.\mathrm{id} \times \alpha_{-\sigma}\right) \cup \eta \cup\left(\mathrm{id} \times \alpha_{\sigma}\right)}{\rightarrow} D_{-}^{2} \times S^{2 n-1} \cup_{\rho} W \cup D^{2} \times \Sigma_{-\sigma}^{2 n-1} \\
=D_{-}^{2} \times S^{2 n-1} \cup_{\rho} D_{+}^{2} \times S^{2 n-1}=\partial \nu_{n+1}\left(C P^{1}\right) .
\end{gathered}
$$

From the next lemma to the effect that two non-isomorphic sphere bundles over $S^{2}$ cannot be PL homeomorphic it follows that the existence of the map $\varphi_{\sigma}$ itself guarantees that $\xi$ and $\partial \nu_{n+1}\left(C P^{1}\right)$ are the same bundle.

LEMMA. For $m \geq 3$ the unique non-trivial orthogonal $S^{m}$ bundle over $S^{2}$, $\xi$, is not PL homeomorphic to $S^{2} \times S^{m}$. 
Proof. Suppose $t: \xi \rightarrow S^{2} \times S^{m}$ is a PL homeomorphism. Let $E$ be the non-trivial $D^{m+1}$ bundle over $S^{2}$ with $\partial E=\xi$ and define the PL manifold

$$
M^{m+3}=E \cup_{t} D^{3} \times S^{m}
$$

$M$ is the union of simply connected spaces over a path connected intersection. Hence, $\pi_{1}(M)=\{1\}$. For $m \geq 3$ the homotopy exact sequence of the fibration $S^{m} \rightarrow \partial E \stackrel{p}{\rightarrow} S^{2}$ implies that $p_{*}: \pi_{2}(\partial E) \rightarrow \pi_{2}\left(S^{2}\right)$ is an isomorphism, and by the Whitehead theorem so is the inclusion $H_{2}(\partial E) \rightarrow H_{2}(E)$. Hence, in the Mayer-Vietoris sequence

$$
\begin{aligned}
\cdots & \rightarrow H_{j}\left(S^{2} \times S^{m}\right) \stackrel{\psi_{j}}{\rightarrow} H_{j}(E) \oplus H_{j}\left(D^{3} \times S^{m}\right) \rightarrow H_{j}(M) \\
& \rightarrow H_{j-1}\left(S^{2} \times S^{m}\right) \rightarrow \cdots
\end{aligned}
$$

$\psi_{j}$ is an isomorphism for $j \leq m+1$. Trivally, $H_{m+2}(M)=0$, and again we have an $(m+2)$-connected $(m+3)$-dimensional PL manifold which is consequently a PL sphere.

Then, $E \cup_{t} D^{3} \times S^{m} \cong S^{m+3}$ defines the vector bundle $E$ as a PL normal micro-bundle to the embedding of its zero section $S^{2} \hookrightarrow S^{m+3}$. By Zeeman's PL unknotting theorem and the uniqueness [7] of stable PL normal microbundles, we see that $E$ and $S^{2} \times D^{m+1}$ must be micro-bundle isomorphic. Let $S^{2} \stackrel{b}{\rightarrow}$ BO classify $E$ as a vector bundle. Then $S^{2} \rightarrow \vec{h}$ BO $\rightarrow \mathrm{BPL}$ is trivial, and as by smoothing theory the fiber PL/0 is 6 -connected we see that $\mathrm{b}$ is homotopically trivial. As $E$ was assumed non-trivial as a vector bundle the PL homeomorphism $t$ cannot exist.

Thus, we define

$$
\varphi_{\sigma}: \partial \nu_{n+1}\left(C P^{1}\right)=\zeta \rightarrow \partial \nu_{n+1}\left(C P^{1}\right) \text { from (1) as required. }
$$

Next we show that the $\varphi_{\sigma}$ just constructed is indeed a self-knotting and that it will suffice for Theorem A.

Recalling from bundle theory that every $S^{N}$ bundle over $S^{2}$ for $N \geq 2$ has a section, we show

Proposition. Any orientation preserving PL homeomorphism $\varphi: \nu \rightarrow \nu$, $\nu$ an orthogonal $S^{N}$ bundle over $S^{2}$, which embeds a section $S^{2} \stackrel{J}{\hookrightarrow} \nu$ homotopically to itself is homotopic to the identity.

Proof. A tubular neighborhood of the section $j\left(S^{2}\right)$ is a $D^{N}$ bundle $U$ in the same stable bundle class as $\nu . \varphi(U)$ PL embeds this bundle in $\nu$ with an inherited smooth structure. By the main theorem of smoothing 
theory ([8] or [13], Thm. 7.3) and the uniqueness of smoothings on $S^{2}$ we can piecewise differentially isotope this embedding to a smooth embedding of $U \rightarrow \nu$. We may easily make the isotopy ambient. Next, we smoothly unknot the core sphere of $U$ and apply the smooth tubular neighborhood theorem. We have, therefore, P.D. isotoped $\varphi$ so that restricted to $U$ it is a $D^{N}$ bundle isomorphism. Since $\pi_{2}(\operatorname{SO}(N))=0$ we can isotope this bundle mapping to the identity through bundle isomorphisms on $U$ all of which extend to $\nu$ as $U$ is a sub-bundle. Thus, we have isotoped $\varphi$ so that it is the identity on $U$. Now, $\nu-U \cong U$ as each fiber of $U$ is a hemisphere of a fiber in $\nu$. We isotope $\varphi \operatorname{rel}(U)$ so that it is the identity on the zero section of the bundle $\nu-U$. Finally, we homotope $\varphi$ to the identity by collapsing the fibers of $\nu-U$ to the zero-section.

We observe that the $\varphi_{\sigma}$ constructed above satisfies the hypothesis of this last proposition as follows: $\varphi_{\sigma}$ is orientation preserving by construction. Also, as the original Brieskorn knot embedded a fiber $S^{2 n+1}$ homotopically to the usual embedding, we know that $\varphi_{\sigma}$ does also. That is $\left(\varphi_{\sigma}\right)_{*}[\partial \nu]=[\partial \nu]$ and $\left(\varphi_{\sigma}\right)^{*}\left(e^{2 n-1}\right)=e^{2 n-1}$, where $e^{2 n-1} \in H^{2 n-1}(\partial \nu)$ is the class represented by inclusion of a fiber. By Poincare Duality, then, $\left(\varphi_{\sigma}\right)_{*}\left(e_{2}\right)=e_{2}$ for $e_{2} \in H_{2}(\partial \nu)$ the class dual to $e^{2 n-1}$. This implies by the Hurewicz Theorem that $\varphi_{\sigma}$ induces the identity homomorphism on $\pi_{2}(\partial \nu)$, which is generated by the inclusion of a section.

The map $\varphi_{\sigma}$ constructed in section C embeds a fiber $S^{2 n-1}$ onto the image of the Brieskorn knot. Hence, in the decomposition

$$
\widetilde{C P}^{n+1}=\left[C P^{n+1}-\nu_{n+1}\left(C P^{1}\right)\right] \cup_{\varphi_{o}}\left[\nu_{n+1}\left(C P^{1}\right)\right]
$$

the identification is in the order:

$$
\varphi_{\sigma}: \partial\left[C P^{n+1}-\nu\right] \rightarrow \partial \nu .
$$

To show, therefore, that $\widetilde{C P}^{n+1} \leftrightarrow(0, \ldots, 0, \sigma)$ we must extend $\varphi_{\sigma}^{-1}$ to a homotopy equivalence $\overline{\varphi_{\sigma}^{-1}}: \nu \rightarrow \nu$ with transverse-inverse image of a fiber being the Milnor or Kervaire manifold $M_{0}^{2 n}$. Note that any extension will be a homotopy equivalence as $\nu \simeq S^{2}$ and $\varphi_{\sigma}^{-1}$ induces the identity on $\pi_{2}(\partial \nu)=\pi_{2}(\nu)$.

Proposition. The PL homeomorphism $\varphi_{\sigma}^{-1}: \partial v_{N+1}\left(C P^{1}\right) \rightarrow \partial v_{n+1}\left(C P^{1}\right)$ constructed above extends to $\bar{\varphi}_{\sigma}^{-1}: \nu_{n+1}\left(C P^{1}\right) \rightarrow \nu_{n+1}\left(C P^{1}\right)$ with transverse-inverse image

$$
\left(\bar{\varphi}_{\sigma}^{-1}\right)^{-1}\left(D^{2 n}\right)=M_{0}^{2 n}
$$


Proof. $\left(\varphi_{\sigma}^{-1}\right)^{-1}\left(S^{2 n-1}\right)=\varphi_{\sigma}\left(S^{2 n-1}\right)=\Sigma_{\sigma}^{2 n-} \subset \partial \nu$ by the construction of $\varphi_{\sigma}$. Moreover, the restriction $\varphi_{\sigma}^{-1} \mid: D^{2} \times \Sigma_{\sigma}^{2 n-1} \rightarrow D_{+}^{2} \times S^{2 n-1}$ is a product map. Now, $\Sigma_{\sigma}^{2 n-1}$ bounds a fiber $F^{2 n} \subset W^{2 n+1}$ whose other boundary component is a fiber $S^{2 n-1}$ of $\partial \nu$. Let $D^{2 n} \subset \nu$ be the fiber whose boundary is this same sphere. Then, $F^{2 n} \cup D^{2 n}=M_{0}^{2 n}$ by the definition of $F^{2 n}$. By pushing $F^{2 n}$ into $\nu$ along a vector field normal to $\partial \nu$ and smoothing the corner at $S^{2 n-1}$ between $F^{2 n}$ and $D^{2 n}$ we obtain a smooth embedding $M_{0}^{2 n} \hookrightarrow \nu$ extending

$$
\partial M_{0}^{2 n}=\Sigma_{\sigma}^{2 n-1} \subset \partial \nu .
$$

Moreover, this embedding will have trivial normal $D^{2}$ bundle as $H^{1}\left(M_{0}^{2 n}, Z\right)=0$. Hence, we can extend the product map

$$
\varphi_{\sigma}^{-1}: D^{2} \times \Sigma_{\sigma}^{2 n-1} \rightarrow D_{+}^{2} \times S^{2 n-1}
$$

to a bundle map $\hat{\varphi}_{\sigma}^{-1}: D^{2} \times M_{0}^{2 n} \rightarrow D_{+}^{2} \times D^{2 n}$ covering a degree one extension $M_{0}^{2 n} \rightarrow D^{2 n}$. Since $\left[\nu-D_{+}^{2}\right] \times D_{-}^{2} \times D^{2 n}=D^{2 n-2}$ there are no cohomology obstructions to extending

$$
\varphi_{\sigma}^{-1} \cup \hat{\varphi}_{\sigma}^{-1} \text { to } \overline{\varphi_{\sigma}^{-1}}: \nu \rightarrow \nu
$$

with the required transverse-inverse image built in.

\section{REFERENCES}

[1] Y. S. Akbulut, Algebraic Equations for a Class of P. L. Manifolds, Ph.D. Thesis, Berkeley, 1974.

[2] Y. S. Akbulut, and H. C. King, Real algebraic variety structures on PL manifolds, Bull. Amer. Math. Soc., 83 (1977), 281-282.

[3] W. Browder, Manifolds with $\pi_{1}=Z$, Bull. Amer. Math. Soc., 72 (1966), 238-244.

[4] __ Surgery on Simply-Connected Manifolds, Springer-Verlag, New York, 1972.

[5] G. Brumfiel, I. Madsen, and R. J. Milgram, PL characteristic classes and cobordism, Annals of Math., 97 (1973), 82-159.

[6] A. Haefliger, Plongemonts differentiables de varietés dans varietés, Comm. Math. Helv., 36 (1961), 47-82.

[7] A. Haefliger and C. T. C. Wall, Piecewise linear bundles in the stable range, Topology, 4 (1965), 209-214.

[8] M. W. Hirsch and B. Mazur, Smoothings of Piecewise Linear Manifolds, Princeton, 1974.

[9] F. Hirzebruch, Singularities and Exotic Spheres, Seminar Bourbaki No. 314, 1966.

[10] M. Kato, A concordance classification of PL homeomorphisms of $S^{p} \times S^{q}$, Topology, 8 (1969), 371-383.

[11] M. Kervaire and J. Milnor, Groups of homotopy spheres I, Annals of Math., 77 (1963), 504-537.

[12] S. Lang, Introduction to Differentiable Manifolds, Interscience, New York, 1962.

[13] R. Lashof and M. Rothenberg, Microbundles and Smoothing, Topology, 3 (1965), 357-388. 
[14] I. Madsen and R. J. Milgram, The oriented topological and PL cobordism rings, Bull. Amer. Math. Soc., 80 (1974), 855-860.

[15] _ The Classifying Spaces for Surgery and Cobordism of Manifolds, Princeton University Press, Princton New Jersey, 1979.

[16] D. Meadows, Some groups of PL self-knotting, Houston J. Math., (to appear).

[17] J. Milnor, Singular Points of Complex Hypersurfaces, Princeton University Press, Princeton, New Jersey, 1968.

[18] J. Milnor and J. D. Stasheff, Characteristic Classes, Princeton University Press, Princeton, New Jersey, 1974.

[19] J. Munkres, Elementary Differential Topology, Princeton, 1966.

[20] C. P. Rourke and B. J. Sanderson, Block bundles I, II, and III, Ann. of Math., 87 (1968), 1-28, 256-278, 431-483.

[21] ___ Introduction to Piecewise-Linear Topology, Springer-Verlag, New York, 1972.

[22] S. Smale, Differentiable and combinatorial structures on manifolds, Ann. of Math., 74 (1961), 498-502.

[23] D. Sullivan, Triangulating and smoothing homotopy equivalences, mimeographed notes, Princeton University.

[24] C. T. C. Wall, Surgery on Compact Manifolds, Academic Press, New York, 1970.

[25] G. W. Whitehead, Homotopy Theory, M.I.T. Press, Cambridge, Massachusetts, 1966.

[26] R. E. Williamson Jr., Cobordism of combinatorial manifolds, Ann. of Math., 83 (1966), 1-133.

[27] H. Winkelnkemper, Manifolds as open books, Bull. Amer. Math. Soc., 79 (1973), 45-51.

[28] E.-C. Zeeman, Seminar on combinatorial topology, I.H.E.S., 1963.

Received March 24, 1980.

UNIVERSITY OF ROCHESTER

ROCHESTER, NY 14627 


\section{PACIFIC JOURNAL OF MATHEMATICS}

\section{EDITORS}

Donald BabbitT (Managing Editor)

University of California

Los Angeles, CA 90024

Hugo Rossi

University of Utah

Salt Lake City, UT 84112

C. C. Moore and Arthur Ogus

University of California

Berkeley, CA 94720
J. DugunduI

Department of Mathematics

University of Southern California

Los Angeles, CA 90089-1113

R. FinN and H. SAmelson

Stanford University

Stanford, CA 94305

\section{ASSOCIATE EDITORS}
R. ARENS
E. F. BECKENBACH
B. H. NEUMANN
F. WOLF
K. YosHIDA (1906-1982)

\section{SUPPORTING INSTITUTIONS}

UNIVERSITY OF ARIZONA

UNIVERSITY OF BRITISH COLUMBIA

CALIFORNIA INSTITUTE OF TECHNOLOGY

UNIVERSITY OF CALIFORNIA

MONTANA STATE UNIVERSITY

UNIVERSITY OF NEVADA, RENO

NEW MEXICO STATE UNIVERSITY

OREGON STATE UNIVERSITY
UNIVERSITY OF OREGON

UNIVERSITY OF SOUTHERN CALIFORNIA

STANFORD UNIVERSITY

UNIVERSITY OF HAWAII

UNIVERSITY OF TOKYO

UNIVERSITY OF UTAH

WASHINGTON STATE UNIVERSITY

UNIVERSITY OF WASHINGTON 


\section{Pacific Journal of Mathematics}

Vol. 107, No. $1 \quad$ January, 1983

John Kelly Beem and Phillip E. Parker, Klein-Gordon solvability and the

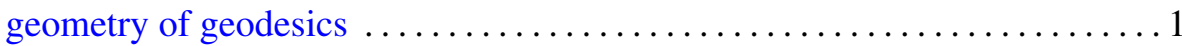

David Borwein and Amnon Jakimovski, Transformations of certain

sequences of random variables by generalized Hausdorff matrices ..... 15

Willy Brandal and Erol Barbut, Localizations of torsion theories . . . . . . . 227

John David Brillhart, Paul Erdős and Richard Patrick Morton, On sums

of Rudin-Shapiro coefficients. II ........................... 39

Martin Lloyd Brown, A note on tamely ramified extensions of rings $\ldots \ldots \ldots 71$

Chang P'ao Ch'ên, A generalization of the Gleason-Kahane-Żelazko

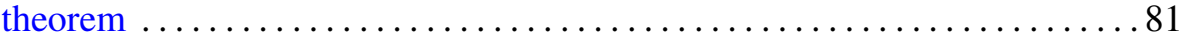

I. P. de Guzman, Annihilator alternative algebras $\ldots \ldots \ldots \ldots \ldots \ldots$. . . . 89

Ralph Jay De Laubenfels, Extensions of $d / d x$ that generate uniformly

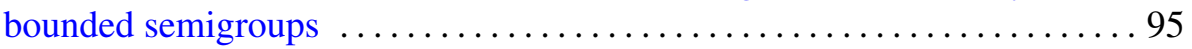

Patrick Ronald Halpin, Some Poincaré series related to identities of $2 \times 2$

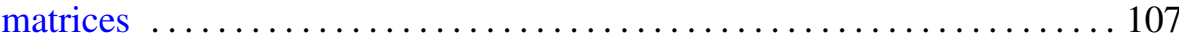

Fumio Hiai, Masanori Ohya and Makoto Tsukada, Sufficiency and

relative entropy in $*$-algebras with applications in quantum systems . . . 117

Dean Robert Hickerson, Splittings of finite groups $\ldots \ldots \ldots \ldots \ldots \ldots \ldots 14$

Jon Lee Johnson, Integral closure and generalized transforms in graded

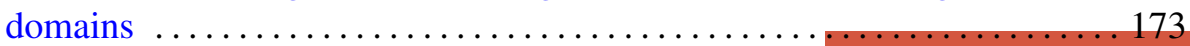

Maria Grazia Marinari, Francesco Odetti and Mario Raimondo, Affine

curves over an algebraically nonclosed field . ................. 179

Douglas Shelby Meadows, Explicit PL self-knottings and the structure of

PL homotopy complex projective spaces $\ldots \ldots \ldots \ldots \ldots \ldots \ldots \ldots \ldots$

Charles Kimbrough Megibben, III, Crawley's problem on the unique

$\omega$-elongation of $p$-groups is undecidable .................... 205

Mary Elizabeth Schaps, Versal determinantal deformations $\ldots \ldots \ldots \ldots 213$

Stephen Scheinberg, Gauthier's localization theorem on meromorphic

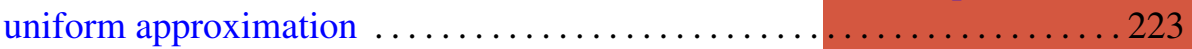

Peter Frederick Stiller, On the uniformization of certain curves . . . . . . . 229

Ernest Lester Stitzinger, Engel's theorem for a class of algebras . . . . . . . . 245

Emery Thomas, On the zeta function for function fields over $F_{p} \ldots \ldots \ldots 251$ 